\title{
Four new isoflavanones from Tadehagi triquetrum
}

\author{
Rong-Ting ZhANG, ${ }^{\text {a,b }}$ Gui-Guang CHENG, ${ }^{a}$ Tao Feng, ${ }^{a}$ Xiang-Hai CAI, ${ }^{a}$ and Xiao-Dong LuO ${ }^{\mathrm{a}, *}$ \\ ${ }^{a}$ State Key Laboratory of Phytochemistry and Plant Resources in West China, Kunming Institute of Botany, Chinese \\ Academy of Sciences, Kunming 650201, China \\ ${ }^{\mathrm{b}}$ Guangxi Botanical Garden of Medicinal Plants, 189 Changgang Road, Nanning 530023, China
}

Received 19 November 2011; Accepted 8 December 2011

(C) The Author(s) 2011. This article is published with open access at Springerlink.com

\begin{abstract}
Four new isoflavanones with isoprenoid units, named triquetrumones E-H (1-4), were isolated from the whole plants of Tadehagi triquetrum. The structures were elucidated on the basis of spectroscopic analyses, including application of MS, UV, IR, 1D and 2D NMR spectroscopic techniques.
\end{abstract}

Keywords: Tadehagi triquetrum, isoflavanone, triquetrumone

\section{Introduction}

Tadehagi triquetrum (Linn.), belonging to the family of Papilionaceae, is an endemic shrub widely distributed in the southern area of Yunnan Province, China. As a traditional DAI medicine in southwest of China, it has been widely used for the treatments of anthelminthic, stomachic, antimicrobial, and inflammation. In addition, it also has been used as a nutrientand appetitive feedstuff. ${ }^{1,2}$

Previous phytochemical studies found a few of isoflavones and phenols from this species. ${ }^{3,4}$ As a part of our continuing research on medicinal plants of DAI ethnopharmacy, ${ }^{6-10}$ we investigated the chemical constituents of the whole plants of $T$. triquetrum, which led to the isolation of four new isoflavanones, named triquetrumones $\mathrm{E}-\mathrm{H}$ (1-4). Isoflavanones possessing isoprenoid unit, can be considered as the characteristic constituents in this plant. This paper describes the isolation and the structural elucidation of these four new compounds.

\section{Results and Discussion}

Compound $\mathbf{1}$ was obtained as yellow amorphous powder which showed an orange fluorescence under UV at $365 \mathrm{~nm}$. The molecular formula was determined as $\mathrm{C}_{27} \mathrm{H}_{26} \mathrm{O}_{7}$ by HRFABMS $\left([\mathrm{M}+\mathrm{H}]^{+}\right.$at $m / z$ 463.1738). The IR spectrum showed bands at $3440(\mathrm{OH}), 1637(\mathrm{C}=\mathrm{O})$, and 1604 (aromatic ring) $\mathrm{cm}^{-1}$. In the ${ }^{1} \mathrm{H}$ NMR spectrum (Table 1), one singlet at $\delta_{\mathrm{H}} 8.03(\mathrm{~s}, \mathrm{H}-2)$, an aromatic C-methyl group at $\delta_{\mathrm{H}} 2.12(\mathrm{~s}, \mathrm{H}-$ 11), two phenolic hydroxyl at $\delta_{\mathrm{H}} 12.62$ (br. s) and 8.05 (br. s), one methoxyl group at $\delta_{\mathrm{H}} 3.84$ (s) and two 2,2-dimethylpyran rings at $\delta_{\mathrm{H}} 5.62\left(\mathrm{~d}, J=9.9, \mathrm{H}-5^{\prime \prime}\right), 6.71\left(\mathrm{~d}, J=9.9, \mathrm{H}-4^{\prime \prime}\right), 1.48$ (s, H-7",8") and $5.63\left(\mathrm{~d}, J=9.9, \mathrm{H}-5^{\prime \prime \prime}\right), 6.82(\mathrm{~d}, J=9.9, \mathrm{H}-$

\footnotetext{
*To whom correspondence should be addressed. E-mail: xdluo@mail.kib.ac.cn
}

4"'), 1.50 (s, Me-7"' and Me-8"') were observed. In addition, the ${ }^{13} \mathrm{C}$ NMR spectrum (Table 2) displayed 15 skeletal $\mathrm{C}$ atoms: one carbonyl at $\delta_{\mathrm{C}} 181.9,13$ quaternary $\mathrm{C}$-atoms at $\delta_{\mathrm{C}}$ 104.7-159.1, and as well one $\mathrm{CH}$ group at $\delta_{\mathrm{C}} 154.7$. Considering the above NMR data and the orange fluorescence under UV at $365 \mathrm{~nm}$, the structure was proposed to be an isoflavone, possessing two isoprenoids units. ${ }^{3}$

The HMBC correlations (Fig. 1) of H-5"/C-8 and H-4" with C-6", C-7, and C-9, and of H-5"'/C-3', and H-4"' with C-6"' and $\mathrm{C}-4$ ' suggested that two dimethylpyrano rings were condensed to $\mathrm{C}-7 / 8$ and $\mathrm{C}-4^{\prime} / 3^{\prime}$, respectively. A methyl at $\delta_{\mathrm{H}} 2.12$ was assigned to connection with C-6 by its correlations with C-5, 6 , 7 in the HMBC spectrum. Moreover, two $\mathrm{OH}$ groups were placed at $\mathrm{C}-5$ and $\mathrm{C}-2$ ', respectively, and a methoxyl group was positioned at $\mathrm{C}^{\prime} 5^{\prime}$ by $\mathrm{HMBC}$ experiments. Complete analysis of the ${ }^{1} \mathrm{H}$ and ${ }^{13} \mathrm{C}$ NMR, HSQC, HMBC data established the structure of $\mathbf{1}$ as $\left(5,2^{\prime}\right.$-dihydroxy-5'-methoxyl6-methyl[6",6"-dimethylpyrano-(2",3":7,8)]-[6"',6"'-dimethylpyrano( $\left.\left(2^{\prime \prime}, 3^{\prime \prime \prime}: 4^{\prime}, 3^{\prime}\right)\right]$-isoflavanone, and named triquetrumone $\mathrm{E}$.

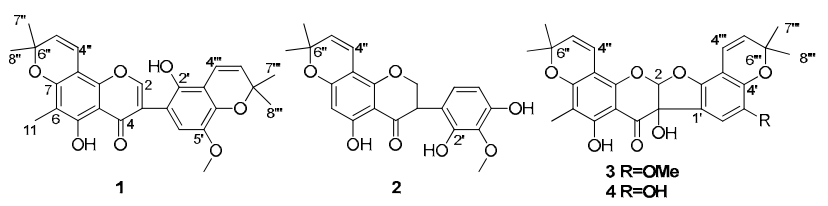

Compound 2 was isolated as yellow amorphous powder. It gave a molecular formula $\mathrm{C}_{21} \mathrm{H}_{20} \mathrm{O}_{7}$ by HRFABMS $\left([\mathrm{M}+\mathrm{H}]^{+}\right.$ at $\mathrm{m} / \mathrm{z}$ 385.1274). Bands for $\mathrm{OH}$ and carbonyl functional groups were assumed by IR absorption at 3421 and 1647 and UV absorptions at $270 \mathrm{~nm}$ were typical for an isoflavanone. ${ }^{11}$ The NMR spectra of compound 2 (Tables 1 and 2) were similar to those of 1"',2"'-dehydrocyclokievitone, ${ }^{12}$ except for the appearance of a methyoxyl group $\left(\delta_{\mathrm{H}} 3.78, \delta_{\mathrm{C}} 60.7\right)$ in $\mathbf{2}$, which was also supported by its molecular formula. The 
Table $1 .{ }^{1} \mathrm{H}$ NMR data of $1-4$.

\begin{tabular}{|c|c|c|c|c|}
\hline position & $1^{\mathrm{a}}$ & $2^{b}$ & $3^{\mathrm{c}}$ & $4^{b}$ \\
\hline 2 & $8.03, \mathrm{~s}$ & $4.53, \mathrm{dd}(5.5,10.9) ; 4.64, \mathrm{t}(10.9)$ & $6.32, \mathrm{~s}$ & $6.29, \mathrm{~s}$ \\
\hline 3 & & $4.27, \mathrm{dd}(10.8,5.5)$ & & \\
\hline 6 & & $5.88, \mathrm{~s}$ & & \\
\hline 11 & $2.12, \mathrm{~s}$ & & $1.92, \mathrm{~s}$ & $1.92, \mathrm{~s}$ \\
\hline $5^{\prime}$ & & $6.38, \mathrm{~d}(8.4)$ & & \\
\hline $6^{\prime}$ & $6.57, \mathrm{~s}$ & $6.69, \mathrm{~d}(8.4)$ & $6.89, \mathrm{~s}$ & $6.78, \mathrm{~s}$ \\
\hline $4 "$ & $6.71, \mathrm{~d}(9.9)$ & $6.51, \mathrm{~d}(10.1)$ & $6.46, \mathrm{~d}(9.9)$ & $6.57, \mathrm{~d}(10.0)$ \\
\hline $5 "$ & $5.62, \mathrm{~d}(9.9)$ & $5.60, \mathrm{~d}(10.1)$ & $5.77, \mathrm{~d}(9.9)$ & $5.67, \mathrm{~d}(10.0)$ \\
\hline $7 "$ & $1.48, \mathrm{~s}$ & $1.41, \mathrm{~s}$ & $1.38, \mathrm{~s}$ & $1.38, \mathrm{~s}$ \\
\hline $8 "$ & $1.48, \mathrm{~s}$ & $1.42, \mathrm{~s}$ & $1.42, \mathrm{~s}$ & $1.41, \mathrm{~s}$ \\
\hline 4"' & $6.82, \mathrm{~d}(9.9)$ & & $6.57, \mathrm{~d}(10.0)$ & $6.46, \mathrm{~d}(9.9)$ \\
\hline $5 " '$ & $5.63, \mathrm{~d}(9.9)$ & & $5.68, \mathrm{~d}(10.0)$ & $5.76, \mathrm{~d}(9.9)$ \\
\hline 7"' & $1.50, \mathrm{~s}$ & & $1.41, \mathrm{~s}$ & $1.38, \mathrm{~s}$ \\
\hline $8^{\prime \prime \prime}$ & $1.50, \mathrm{~s}$ & & $1.45, \mathrm{~s}$ & $1.41, \mathrm{~s}$ \\
\hline $\mathrm{OMe}$ & $3.84, \mathrm{~s}$ & $3.78, \mathrm{~s}$ & $3.73, \mathrm{~s}$ & \\
\hline $\mathrm{OH}(5)$ & 12.62, br. s & 12.44 , br. s & 12.27, br. $\mathrm{s}$ & 12.27, br. s \\
\hline $\mathrm{OH}\left(2^{\prime}\right)$ & 8.05 , br. s & 8.15 , br. s & & \\
\hline $\mathrm{OH}\left(4^{\prime}\right)$ & & 8.30 , br. s & & \\
\hline $\mathrm{OH}\left(5^{\prime}\right)$ & & & & 7.50 , br. s \\
\hline $\mathrm{OH}(3)$ & & & 6.08 , br. s & 6.05 , br. s \\
\hline
\end{tabular}

${ }^{\mathrm{a}}$ Measured in $\mathrm{CDCl}_{3}$ at $400 \mathrm{MHz} ;{ }^{\mathrm{b}}$ Measured in acetone- $d_{6}$ at $500 \mathrm{MHz} ;{ }^{\mathrm{c}}$ Measured in acetone- $d_{6}$ at $400 \mathrm{MHz}$.

methyoxyl group was paced at C-3', supported by the HMBC correlations (Fig. 1). Thus, compound 2 was established to be 5,2',4'-trihydroxy-3'-methoxy[6",6"-dimethylpyrano(2",3":7,8)]isoflavavone, and named triquetrumone $\mathrm{F}$.

Compound $\mathbf{3}$ appeared as yellow amorphous powder whose molecular was established as $\mathrm{C}_{27} \mathrm{H}_{26} \mathrm{O}_{8}$ by HRFABMS at $\mathrm{m} / \mathrm{z}$ 479.1723 (calcd. for $\mathrm{C}_{27} \mathrm{H}_{27} \mathrm{O}_{8}, 479.1706$ ), which indicated 15 degrees of unsaturation. T. triquetrum are known to contain three 5-hydroxy-coumaronochromones, and all of which fluoresce on silica gel thin-layer plates under $365 \mathrm{~nm}$ of UV light. ${ }^{3}$ Take UV, MS, and NMR data into account, compound 3 can be most logically formulated as an isoflavone, in which $\mathrm{C}-2$ and $\mathrm{C}-2^{\prime}$ was linked by an ether oxygen to form a pentacyclic ring system. ${ }^{13}$ The ${ }^{1} \mathrm{H}$ NMR spectrum (Table 1 ) of 3 revealed two 2,2-dimethylpyrane rings at $\delta_{\mathrm{H}} 5.77$ (d, $J=9.9$, H-5"), 6.46 (d, $J=9.9, \mathrm{H}-4 "), 1.38$ (s, Me-7") and 1.42 (s, Me$\left.8^{\prime \prime}\right)$, together with 5.68 (d, $\left.J=10.0, \mathrm{H}-5^{\prime \prime \prime}\right), 6.57$ (d, $J=10.0$, H-4"'), 1.41 (s, Me-7"') and 1.45 (s, Me-8"'), associated with an aromatic C-methyl group at $\delta_{\mathrm{H}} 1.92(\mathrm{~s})$ and two singlets at $\delta_{\mathrm{H}} 6.32(\mathrm{~s}, \mathrm{H}-2)$ and $6.89(\mathrm{~s}, \mathrm{H}-6$ '), respectively.

The ${ }^{13} \mathrm{C}$ and ${ }^{1} \mathrm{H}$ NMR spectra of 3 implied that this compound might be an analogue of triquetrumone $\mathrm{B}{ }^{3}$ Careful comparison of the NMR data of two compounds revealed that one more $\mathrm{OH}$ group might be appeared at C-3, suggested by downshift signals at $\delta_{\mathrm{C}} 79.4(\mathrm{~s}, \mathrm{C}-3), \delta_{\mathrm{H}} 6.08$ (br. s, OH) in $\mathbf{3}$. The proposal was further supported by the HMBC correlations (Fig. 1) between OH-3 with $\delta_{\mathrm{C}} 193.4$ (s, C-4), 111.5 (d, C-2) and 79.4 (s, C-3). Thus, 3 was established as 3,5-dihydroxy-5'methoxyl-6-methyl[6",6"-dimethylpyrano(2",3":7,8)]-[6"',6"'dimethylpyrano(2"',3"':2',3')]coumaronochromone, and named triquetrumone $\mathrm{G}$.

Compound 4 was obtained as yellow amorphous powder. The HRFABMS clearly showed the $[\mathrm{M}+\mathrm{H}]^{+}$in agreement with the molecular formula $\mathrm{C}_{26} \mathrm{H}_{24} \mathrm{O}_{8}$. The UV, IR, and NMR spectroscopic data also suggested a coumaronochromone structure for $4,{ }^{14}$ which was very similar to those of $\mathbf{3}$. Detailed comparison of the NMR data of two compounds revealed that a hydroxyl group was appeared on ring $\mathrm{C}$ of 4 , instead of the methyoxyl in 3, concurring with its molecular formula. The assignment was also supported by the HMBC correlations (Fig.
1) of $\delta_{\mathrm{H}} 7.50\left(s, \mathrm{OH}-5^{\prime}\right)$ with $\delta_{\mathrm{C}} 106.9\left(\mathrm{~s}, \mathrm{C}-3^{\prime}\right), 142.1$ (s, C-4') and $143.0\left(\mathrm{~s}, \mathrm{C}-5^{\prime}\right)$. Thus, the structure of 4 was established as 3,5,5'-trihydroxy-6-methyl[6",6"-dimethylpyrano( $(2 ", 3 ": 7,8)]$ $\left[6^{\prime \prime}, 6^{\prime \prime}\right.$-dimethypyrano $\left.\left(2^{\prime \prime}, 3^{\prime \prime}: 2^{\prime}, 3^{\prime}\right)\right]$ coumaronochromone, and named triquetrumone $\mathrm{H}$.

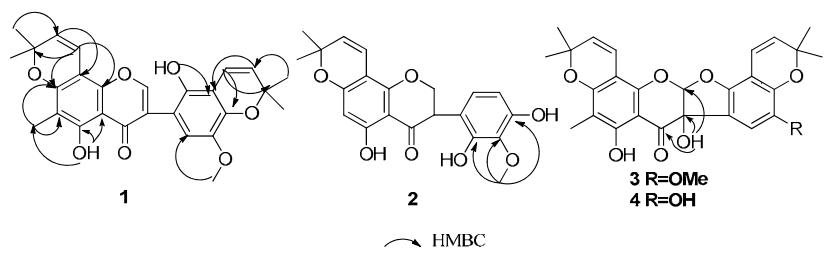

Fig 1. Key HMBC correlations of 1-4.

\section{Experimental Section}

General Experimental Procedures. Column chromatography (CC): Silica gel (200-300 mesh, Qingdao Marine Chemical corporation, China) and Sephadex LH-20 (Amersham Biosciences, Sweden); TLC monitoring, visualization by heating the silica gel plates sprayed with $10 \% \mathrm{H}_{2} \mathrm{SO}_{4}$ in EtOH. Optical rotations: Horiba SEPA-300 polarimeter. UV spectra: Shimadzu 210-A double-beam spectrophotometer; $\lambda_{\max }$ $(\log \varepsilon)$ in nm. IR Spectra: Brucker Tensor 27 spectrometer ; $\mathrm{KBr}$ pellets; in $\mathrm{cm}^{-1}$. NMR Spectra: Bruker AM-500 spectrometers; $\delta$ in ppm with $\mathrm{SiMe}_{4}$ as internal standard, $J$ in $\mathrm{Hz}$. MS: $V G$ Autospec-3000 and API QSTAR-Palsar-I spectrometer.

Plant Material. The whole plants of Tadehagi triquetrum were collected from Xishuangbanna, Yunnan Province, China, in April 2007. Its identity was confirmed by Mr. Jing-Yun Cui, and a voucher specimen (NO. 2007042) has been deposited in the State Key Laboratory of Phytochemistry and Plant Resources in West China, Kunming Institute of Botany, Chinese Academy of Sciences.

Extraction and Isolation. The dried and milled sample (10 $\mathrm{kg})$ were soaked in $95 \% \mathrm{EtOH}(40 \mathrm{~L} \times 3)$ under reflux $(48 \mathrm{~h} \times 3)$, and the solvent was evaporated in vacuo. The

\section{照 Springer}


Table 2. ${ }^{13} \mathrm{C}$ NMR data of 1-4.

\begin{tabular}{|c|c|c|c|c|}
\hline pos. & $1^{\mathrm{a}}$ & $2^{b}$ & $3^{\mathrm{c}}$ & $4^{b}$ \\
\hline 2 & $154.7, \mathrm{CH}$ & $71.2, \mathrm{CH}_{2}$ & $111.5, \mathrm{CH}$ & $111.4, \mathrm{CH}$ \\
\hline 3 & $123.4, \mathrm{C}$ & $47.5, \mathrm{CH}$ & $79.4, \mathrm{C}$ & $79.3, \mathrm{C}$ \\
\hline 4 & $181.9, \mathrm{C}$ & $198.5, \mathrm{C}$ & $193.4, \mathrm{C}$ & $193.6, \mathrm{C}$ \\
\hline 5 & 159.1, C & $165.0, \mathrm{C}$ & $162.4, \mathrm{C}$ & $162.3, \mathrm{C}$ \\
\hline 6 & $109.8, \mathrm{C}$ & $97.6, \mathrm{CH}$ & 107.0, C & 107.1, C \\
\hline 7 & $158.3, \mathrm{C}$ & $162.4, \mathrm{C}$ & $161.4, \mathrm{C}$ & 161.3, C \\
\hline 8 & $100.5, \mathrm{C}$ & $102.3, \mathrm{C}$ & 102.6, C & $102.5, \mathrm{C}$ \\
\hline 9 & $150.1, \mathrm{C}$ & $158.2, \mathrm{C}$ & $152.1, \mathrm{C}$ & $152.7, \mathrm{C}$ \\
\hline 10 & 104.7, C & $103.9, \mathrm{C}$ & $100.7, \mathrm{C}$ & $101.6, \mathrm{C}$ \\
\hline 11 & 7.3, C & & $6.8, \mathrm{CH}_{3}$ & $6.8, \mathrm{C}$ \\
\hline $1^{\prime}$ & $110.8, \mathrm{C}$ & $114.0, \mathrm{C}$ & $117.5, \mathrm{C}$ & $118.2, \mathrm{C}$ \\
\hline $2^{\prime}$ & 146.1, C & $149.6, \mathrm{C}$ & $150.3, \mathrm{C}$ & $148.8, \mathrm{C}$ \\
\hline $3^{\prime}$ & 113.6, C & $136.4, \mathrm{C}$ & 107.6, C & $106.9, \mathrm{C}$ \\
\hline $4^{\prime}$ & $144.5, \mathrm{C}$ & $150.9, \mathrm{C}$ & $145.4, \mathrm{C}$ & $142.1, \mathrm{C}$ \\
\hline $5^{\prime}$ & $142.8, \mathrm{C}$ & $108.3, \mathrm{CH}$ & 146.0, C & $143.0, \mathrm{C}$ \\
\hline $6^{\prime}$ & $114.1, \mathrm{CH}$ & 125.7, $\mathrm{CH}$ & $110.6, \mathrm{CH}$ & $111.7, \mathrm{CH}$ \\
\hline 4" & 114.6, CH & $116.0, \mathrm{CH}$ & $115.8, \mathrm{CH}$ & $115.8, \mathrm{CH}$ \\
\hline $5^{\prime \prime}$ & $129.4, \mathrm{CH}$ & $127.3, \mathrm{CH}$ & $128.1, \mathrm{CH}$ & $128.0, \mathrm{CH}$ \\
\hline 6" & 78.3, C & 78.7, C & 79.4, C & 79.3, C \\
\hline 7" & $28.3, \mathrm{CH}_{3}$ & $28.3, \mathrm{CH}_{3}$ & $28.0, \mathrm{CH}_{3}$ & $27.7, \mathrm{CH}_{3}$ \\
\hline $8^{\prime \prime}$ & $28.3, \mathrm{CH}_{3}$ & $28.5, \mathrm{CH}_{3}$ & $28.7, \mathrm{CH}_{3}$ & $27.8, \mathrm{CH}_{3}$ \\
\hline 4"' & $117.8, \mathrm{CH}$ & & $116.3, \mathrm{CH}$ & $116.3, \mathrm{CH}$ \\
\hline $5^{\prime \prime \prime}$ & $127.5, \mathrm{CH}$ & & 131.6, CH & $131.7, \mathrm{CH}$ \\
\hline 6"' & $76.5, \mathrm{CH}$ & & $77.6, \mathrm{C}$ & 77.8, C \\
\hline 7"' & $27.8, \mathrm{CH}_{3}$ & & $28.6, \mathrm{CH}_{3}$ & $28.5, \mathrm{CH}_{3}$ \\
\hline $8^{\prime \prime \prime}$ & $27.8, \mathrm{CH}_{3}$ & & $29.3, \mathrm{CH}_{3}$ & $28.6, \mathrm{CH}_{3}$ \\
\hline $\mathrm{OMe}$ & $57.4, \mathrm{CH}_{3}$ & $60.7, \mathrm{CH}_{3}$ & $57.5, \mathrm{CH}_{3}$ & \\
\hline
\end{tabular}

${ }^{\mathrm{a}}$ Recorded in $\mathrm{CDCl}_{3}$ at $100 \mathrm{MHz} ;{ }^{\mathrm{b}}$ Recorded in acetone- $d_{6}$ at $100 \mathrm{MHz}$; ${ }^{\mathrm{c}}$ Recorded in acetone- $d_{6}$ at $125 \mathrm{MHz}$.

residue was partitioned between EtOAc and $\mathrm{H}_{2} \mathrm{O}$. The EtOAc extract $(80 \mathrm{~g})$ was subjected to $\mathrm{CC}\left(\mathrm{CHCl}_{3} / \mathrm{Me}_{2} \mathrm{CO} 10: 1 \rightarrow 1: 1\right)$ : eight fractions $(F r .1-8)$ by TLC. Fr.3 $(15 \mathrm{~g})$ was repeatedly subjected to $\mathrm{CC}$ (1. Silica-G petroleum ether/ $\mathrm{Me}_{2} \mathrm{CO}$ $10: 1 \rightarrow 2: 1 ; 2$. Sephadex $\left.\mathrm{LH}-20, \mathrm{CHCl}_{3} / \mathrm{MeOH} 1: 1\right)$ to afford 1 (6 mg). Fr.4 (12 g) was purified by repeated $\mathrm{CC}\left(\mathrm{SiO}_{2}\right.$, petrolrum ether $\left./ \mathrm{Me}_{2} \mathrm{CO} 3: 1\right)$ to afford $2(21 \mathrm{mg})$. Fr.5 (10 g) was resubmitted to $\mathrm{CC}\left(1 . \mathrm{SiO}_{2}, \mathrm{CHCl}_{3} / \mathrm{MeOH} 70: 1 \rightarrow 50: 1 ; 2\right.$. Sephadex $\left.\mathrm{LH}-20, \mathrm{MeOH} / \mathrm{H}_{2} \mathrm{O} 9: 1\right)$ to provide $3(5 \mathrm{mg})$ and 4 (25 mg).

Triquetrumone E (1): yellow amorphous powder, UV $(\mathrm{MeOH}) \lambda_{\max }(\log \varepsilon) 273$ (4.49), $212(4.51) \mathrm{nm}$. IR (KBr) $v_{\max }$ 3440, 2924, 1637, $1604 \mathrm{~cm}^{-1}$. ${ }^{1} \mathrm{H}$ and ${ }^{13} \mathrm{C}$ NMR data see Tables 1 and 2. ESIMS (positive): $463\left([\mathrm{M}+\mathrm{H}]^{+}\right)$. HRFABMS (positive): $463.1738\left([\mathrm{M}+\mathrm{H}]^{+}, \mathrm{C}_{27} \mathrm{H}_{27} \mathrm{O}_{7}\right.$, calcd. 463.1757).

Triquetrumone F (2): yellow amorphous powder, $[\alpha]_{\mathrm{D}}^{20}$ -1.63 (c 0.002, $\left.\mathrm{Me}_{2} \mathrm{CO}\right)$. UV (MeOH) $\lambda_{\max }(\log \varepsilon) 270$ (4.24), 207 (4.42) nm. IR (KBr) $v_{\max } 3421,1647,1598 \mathrm{~cm}^{-1} .{ }^{1} \mathrm{H}$ and ${ }^{13} \mathrm{C}$ NMR data see Tables 1 and 2. ESIMS (positive): 385 ([M $\left.+\mathrm{H}]^{+}\right)$. HRFABMS (positive): $385.1274\left([\mathrm{M}+\mathrm{H}]^{+}, \mathrm{C}_{21} \mathrm{H}_{21} \mathrm{O}_{7}\right.$, calcd. 385.1287).

Triquetrumone $\mathbf{G}$ (3): yellow amorphous powder, $[\alpha]_{\mathrm{D}}^{20}$ -44.5 (c 0.001, $\left.\mathrm{Me}_{2} \mathrm{CO}\right)$. UV(MeOH) $\lambda_{\max }(\log \varepsilon) 273$ (4.01), 268 (4.02), $212(4.39) \mathrm{nm}$. IR (KBr) $v_{\max } 3441,1632,1600$ $\mathrm{cm}^{-1}$. ${ }^{1} \mathrm{H}$ and ${ }^{13} \mathrm{C}-\mathrm{NMR}$ data see Tables 1 and 2. EIMS: 478
$[\mathrm{M}]^{+}$. HRFABMS (positive): $479.1723\left([\mathrm{M}+\mathrm{H}]^{+}, \mathrm{C}_{27} \mathrm{H}_{27} \mathrm{O}_{8}\right.$, calcd. 479.1706).

Triquetrumone $\mathbf{H}$ (4): yellow amorphous powder, $[\alpha]_{\mathrm{D}}^{20}$ -32.3 (c $\left.0.001, \mathrm{Me}_{2} \mathrm{CO}\right)$. UV $(\mathrm{MeOH}) \lambda_{\max }(\log \varepsilon) 274(4.34)$ 268 (4.34), $212(4.45) \mathrm{nm}$. IR (KBr) $v_{\max } 3424,1635,1599$ $\mathrm{cm}^{-1} \cdot{ }^{1} \mathrm{H}$ and ${ }^{13} \mathrm{C}$ NMR data see Tables 1 and 2. EIMS: 464 $[\mathrm{M}]^{+}$. HRFABMS (positive): $465.1556\left([\mathrm{M}+\mathrm{H}]^{+}, \mathrm{C}_{26} \mathrm{H}_{25} \mathrm{O}_{8}\right.$, calcd. 465.1549).

\section{Electronic Supplementary Material}

Supplementary material is available in the online version of this article at http://dx.doi.org/10.1007/s13659-011-0033-5 and is accessible for authorized users.

\section{Acknowledgments}

The authors are grateful to National Basic Research Program of China (973 Program 2009CB522300), and the Chinese Academy of Science (KSCX2-EW-R-15) for financial support and to the members of the analytical group in the State Key Laboratory of Phytochemistry and Plant Resources in West China, Kunming Institute of Botany, Chinese Academy of Sciences, for the spectral measurements.

Open Access This article is distributed under the terms of the Creative Commons Attribution License which permits any use, distribution, and reproduction in any medium, provided the original author(s) and source are credited.

\section{References}

[1] ZhonghuaBencao Gangyao daiyaojuan, Shanghai Science and Technology Press: Shanghai, 2005, p. 52.

[2] Xishuangbanna Daiyaozhi. Xishuangbanna health bureau Press: Xishuangbanna, 1979, p. 116.

[3] Xiang, W; Li, R. T.; Mao, Y. L.; Zhang, H. J.; Li, S. H.; Song, Q. S.; Sun, H. D. J. Agric. Food Chem. 2005, 53, 267-271.

[4] Wen, D. X.; Zheng, X. Z.; Shi, J. X. Chin. Tradit. Herb. Drugs 1999, 30, 252-253.

[5] Cai, X. H.; Du Z. Z.; Luo, X. D. Org. Lett. 2007, 9, 1817-1820.

[6] Feng, T.; Cai, X. H.; Zhao, P. J.; Du Z. Z.; Li, W. Q.; Luo, X. D. Planta Med. 2009, 75, 1537-1541.

[7] Feng, T.; Xu, Y; Cai, X. H.; Du Z. Z.; Luo, X. D. Planta Med. 2009, 75, 76-79.

[8] Cai, X. H.; Feng, T; Liu, Y. P.; Du Z. Z.; Luo, X. D. Org. Lett. 2008, 10, 577-580.

[9] Liu, Y. P.; Cai, X. H.; Du Z. Z.; Li, W. Q.; Luo, X. D. Z. Naturforsch., B 2008, 915-919.

[10] Liu, Y. P.; Cai, X. H.; Li, W. Q.; Luo, X. D. Helv. Chim. Acta 2008, 91, 1299-1304.

[11] Mabry, T. J.; Markham, K. J.; Thomas, M. B. The Systematic Identification of Flavonoids, Springer-Verlag, New York, 1970.

[12] Woodward, M. D. Phytochemistry 1979, 18, 2007-2010.

[13] Tahara, S.; Ingham, J. L.; Mizutani, J. Agric. Biol. Chem. 1985, $49,1775-1783$

[14] Tahara, S.; Orihara, S.; Ingham, J. L.; Mizutani, J. Phytochemistry 1989, 28, 901-911. 